\title{
Ueber den Nachweis von Cystin und Cystein unter den Spaltungsprodukten der Eiweisskörper.
}

\author{
Von \\ Gustar Embden,
}

zweitem Assistenten am physiologischen Institut der Universität Zürich.

(Aus dem physiologisch-chemischen Institut zu Strassburg. Neue Folge Nr. 43).

(Der Redaction zugegangen am 25. December 1900.)

Dass Cystin als Spaltungsprodukt von Eiweisskörpern auftritt, war schon durch ältere gelegentliche Befunde von R. Külz ${ }^{1}$ ) und Emmerling ${ }^{2}$ ) nahe gelegt worden, doch waren die Bemühungen, es durch Darstellung und Analyse sicher nachzuweisen, vergeblich gewesen, bis es vor etwas mehr als Jahresfrist K. A. H. Mörner ${ }^{3}$ ) in einer wichtigen Untersuchung gelang, die rcichliche Bildung von Cystin bei Hydrolyse von Keratin ausser Frage zu stellen.

Ohne Kenntniss von dieser Untersuchung zu haben, gelangte ich kurze Zeit nach deren Erscheinen auf wesentlich abweichendem Wege zum gleichen Resultat und bei Fortführung der Versuche zu einigen, wie mir schien, beachtenswerthen Befunden.

In einem Vortrag auf dem diesjährigen internationalen Congress in Paris hat Mörner seine früheren Angaben vervollständigt und dabei eine Reihe werthvoller neuer Thatsachen mitgetheilt.

Da der Vortrag wenig bekannt geworden ist, hat mir Herr Professor Mörner gestattet, den wesentlichen Inhalt desselben, soweit er auf vorliegende Arbeit Bezug hat, auf Grund einer freundlichen brieflichen Mittheilung hier in Kürze anzuführen:

1) Zeitschrift für Biologie, Bd. 27, S. 415.

2) Chemikerzeitung 1894, S, 1539.

3) Zeitschrift für physiol. Chemie, Bd. XXVIII, S. 595. 
Mörner verbesserte und vereinfachte sein Verfahren der Cystindarstellung dadurch, dass er nach dem Zerlegen mit Salzsäure und nach Abdestilliren der Hauptmenge der Säure den Rückstand in schwachem Weingeist löste, mit Natronlauge oder Ammoniak (statt wie früher mit Bleioxyd) neutralisirte und den Niederschlag auf Cystin verarbeitete. Diese Darstellungsmethode benutzte er weiter zur approximativen Bestimmung des Cystins und neben gleichzeitiger Ermittelung des Gesammtschwefels und des bleischwärzenden Schwefels zum Studium der Bindungsweise des Schwefels in den Keratinsubstanzen.

Er vermochte auf dem eingeschlagenen Wege reichlich Cystin ausser aus Horn auch aus der Schaalenhaut des Hühnereies und aus Menschenhaaren rein zu gewinnen. Er beobachtete dabei, dass die Bildung von Cystin nicht nothwendig mit jener von Cystein einhergeht, und ist jetzt mehr noch, als aus seiner ersten Publication hervorging, geneigt, die Entstehung von Cystein als einen secundären Vorgang anzusehen.

Die approximativen Bestimmungen des Cystins, deren Ergebniss Mörner als eher zu niedrig, denn zu hoch ansieht, ergaben für Hornsubstanz $6,8 \%$, für die Schaalenhaut des Eies $6,0 \%$, für die Substanz aus Menschenhaaren 12,6\% Cystin.

Mörner vermochte ferner auch aus echten Eiweissstoffen Cystin zu gewinnen. Aus dem Serumalbumin erhielt er etwas mehr als $1 \%$ eines in sechsseitigen Täfelchen krystallisirten Präparates, dessen Identität mit Cystin durch die Bestimmung des Schwefelgehalts ausser Zweifel gestellt wurde. ${ }^{1}$ )

Meine einschlägigen, ohne Kenntniss dieser Befunde Mörner's gesammelten Erfahrungen haben ihnen gegenüber

1) Wie ich der brieflichen Mittheilung von Herrn Prof. Mörner entnehme, gelang es ihm seit dem auch, die Angabe von Külz über Bildung von Cystin bei tryptischer Verdauung unter Verwendung von käuflichem Bluteiweiss zu bestätigen. Zum gleichen Resultat ist übrigens auch Herr Dr. E. Petry im hiesigen Institute gelangt, bei Versuchen, welche das Schicksal der schwefelreichen Gruppe des Eiweissmoleküls im Verlauf der tryptischen Spaltung klarstellen sollten. 
zum Theil nur den Werth einer Bestätigung. Trotzdem dürfte deren Mittheilung, zumal die von mir angewandte Methode mehrfach von jener Mörner's abwich, nicht ohne Nutzen sein.

\section{Darstellung von Cystin aus Hornspänen.}

Zur raschen und bequemen Gewinnung von grösseren Mengen Cystin aus Hornspänen hat sich in zum Theil von mir allein, zum Theil gemeinsam mit Herrn Dr. V.Ducceschi ausgeführten Versuchen ein Verfahren bewährt, dessen wesentliche Operationen waren : Zersetzen der Hornspäne durch mehrstündiges Kochen mit concentrirter Salzsäure, Neutralisiren der Zersetzungsflüssigkeit mit Natronlauge, 24 stündiges Stehenlassen, dann Abfiltriren des abgeschiedenen «Melaninniederschlags», Entfärbung des schwach mit Salzsäure angesäuerten Filtrats mit Thierkohle, Einengen zur Krystallisation, $A b-$ trennung der sich ausscheidenden Krystallmassen, Befreiung des darin enthaltenen Cystins von Kochsalz und Leucin mit Wasser, endlich Trennung vom Tyrosin mit Hülfe sehr stark verdünnter Salpetersäure.

W'ie man sieht, weicht dieses Vorgehen von Mörner's ursprünglichem Verfahren (einwöchentliches oder längeres Erhitzen mit Salzsäure auf dem Wasserbad, Trennung des Cystins vom Tyrosin durch fractionirte Krystallisation etc.) in wesentlichen Punkten ab. Dem jüngst mitgetheilten modificirten Verfahren Mörner's steht es jedenfalls näher, ist mit ihm aber, soweit sich beurtheilen lässt, sicher nicht identisch. Als wesentliche Vereinfachung dürfen die Abkürzung der Zersetzungszeit und das Umgehen der Salzsäuredestillation angesehen werden. Wichtig für die rasche Gewinnung reiner Produkte ist auch die Abscheidung der «Melanine», welche anscheinend in der bei der Neutralisation entstehenden concentrirten Salzlösung unlöslich werden.

Die Trennung des Cystins vom Tyrosin mit Hülfe verdünnter Salpetersäure, worin sich ersteres sehr schwer, letzteres sehr leicht löst, führt ungleich rascher zum Ziele, als die von Mörner benutzte fractionirte Krystallisation aus ammoniakhaltigem Wasser. 
Aus der nach dem Kochen mit Thierkohle erhaltenen, wenig gefärbten Lösung fiel, wie nebenbei bemerkt sei, neben Cystin auch das Tyrosin und beim weiteren Einengen das Leucin nahezu farblos aus. Das Cystin schied sich nur selten direkt aus der Mutterlauge in den bekannten sechsseitigen Tafeln ab, sondern bildete zunächst meist Kugeln, wie dies schon von Mörner beschrieben wurde.

Löste man das in Kugeln ausgeschiedene Cystin in verdünntem Ammoniak, so schied es sich bei der spontanen Verdunstung des Ammoniaks oder beim vorsichtigen Ansäuern mit Essigsäure in den charakteristischen sechsseitigen Täfelchen ab. In Nadeln krystallisirtes Cystin, wie es von Mörner beschrieben ist, habe ich aus Horn bisher nicht erhalten; dies ist vielleicht auf die kurze Dauer der Säurespaltung zurückzuführen.

Ueber die Einzelheiten des Vorgehens mag nachstehender Auszug aus den Protokollen Aufschluss geben:

$500 \mathrm{~g}$ käuflicher Hornspäne wurden mit $1500 \mathrm{ccm}$. reiner concentrirter Salzsäure in einem mit Steigrohr versehenem Kolben circa 5-6 Stunden auf dem Sandbade bis zu kräftigem Sieden erhitzt. Die gewonnene, tief schwarzbraune, nur geringe Mengen ungelöster Substanz enthaltende Flüssigkeit wurde nach dem Abkühlen mit concentrirter Natronlauge unter Vermeidung von stärkerem Erwärmen bis zur deutlich amphoteren Reaction versetzt. Nach 24stündigem Stehen wurde von dem entstandenen schwarzbraunen Niederschlag 1) abfiltrirt, und das Filtrat nach schwachem Ansäuern mit Salzsäure durch Kochen mit Thierkohle entfärbt. Das so gewonnene schwachsaure Filtrat war völlig klar und von hellgelber Färbung. Nach 24 stündigem Stehen in der Kälte hatte sich stets ein reichlicher krystallinischer Niederschlag ausgeschieden, der in der Hauptmasse aus Tyrosin bestand, doch oft auch nicht geringe Mengen Cystin enthielt. Nach Abtrennung dieses Niederschlages wurde die Flüssigkeit auf dem Wasserbade ein wenig eingeengt und nach dem Abkühlen von dem neu enstandenen Niederschlage abfiltrirt. Dieses Verfahren wurde einige Male wiederholt, bis die Flüssigkeit auf einige $100 \mathrm{ccm}$. eingeengt war. (Diese letzte Mutterlauge enthielt noch immer viel abspaltbaren Schwefel. Die letzten Krystallisationen sind selbstverständlich immer ausserordentlich kochsalzreich.)

1) Lässt man die mit Natronlauge versetzte Flüssigkeit zu lange stehen, so enthält der Niederschlag unter Umständen recht erhebliche Mengen Tyrosin und auch Cystin.

Hoppe-Seyler's Zeitschrift f. physiol. Chemie. XXXII. 
Die Verarbeitung der einzelnen Krystallisationsfractionen auf Cystin geschah folgendermassen. Sie wurden mit wenig kaltem Wasser versetzt, wobei Kochsalz und eventuell Leucin in Lösung gingen; der abfiltrirte Rückstand löste sich bis auf eine meist geringfügige Menge anorganischer Substanz in wenig verdünntem Ammoniak; nach der spontanen Verdunstung des Ammoniaks oder auf Essigsäurezusatz schied sich ein Gemenge aus, das fast ausschliesslich aus Tyrosin und Cystin bestand und ohne erhebliche Verluste mit ganz kaltem Wasser gewaschen werden konnte.

(Die Vertheilung des Cystins resp. des Tyrosins auf die einzelnen Fractionen war übrigens auffällig verschieden. Schied sich auch gewöhnlich die Hauptmasse des Cystins nach der Hauptmasse des Tyrosins aus, so kam doch auch das Umgekehrte vor.)

Das Gemenge von Cystin und Tyrosin wurde mit einer nicht $z u$ geringen Menge kalten Wassers (etwa auf $5 \mathrm{~g}$ Substanz 100-200 ccm.) versetzt und nun tropfenweise unter stetem Umrühren verdünnte Salpetersäure hinzugefügt. Hat man, was häufig der Fall ist, ein Gemenge von sechsseitigen Cystintafeln mit Tyrosinnadeln, so kann man die Lösung des Tyrosins mikroskopisch verfolgen und so einen unnöthigen Ueberschuss von Salpeteräure thunlichst vermeiden. Das im Rückstand verbleibende Cystin wird durch Waschen mit kaltem Wasser yon der Salpetersäure befreit und nun mehrfach aus Ammoniak in der bekannten Weise umkrystallisirt. Schon nach einmaligem Umkrystallisiren zeigte das Cystin keine Spur Millon'scher Reaction, und andererseits war das Tyrosin, das durch vorsichtiges Neutralisiren der salpetersauren Lösung ausgefällt wurde, frei von abspaltbarem Schwefel.

Die so gewonnenen Cystinpräparate sind in der Regel sehr wenig gefärbt. Behufs weiterer Reinigung wird das Cystin nochmals in salzsaurer Lösung mit Tierkohle behandelt.

Die Ausbeute an Cystin ist bei diesem Verfahren zwar nicht so gross, wie Mörner angibt; bei dem geringen Werth des Ausgangsmaterials wird aber dieser Nachtheil durch die Möglichkeit, grosse Mengen in kurzer Zeit zu verarbeiten, mehr als ausgeglichen.

Gewinnung des Oystins durch Fällung desselben als Kupfersalz.

Für den Nachweis von Cystin erwies sich noch ein zweiter Weg als gangbar. ${ }^{1}$ )

1) Auf diesem Wege bin ich zuerst in der Zersetzungsflüssigkeit des Keratins auf Cystin gestossen und auch Herr Dr. Petry schlng diesen Weg ein, um den Nachweis von Cystin bei Pankreasverdauung zu führen. 
Bei Verarbeitung der Kochsalz, Leucin und andere Aminosäuren enthaltenden Mutterlaugen wurde gelegentlich ein Theil behufs Bildung von Leucinkupfer mit alkalifreiem Cuprihydroxyd versetzt und vorsichtig auf dem Wasserbade unter stetem Umrühren erwärmt. Schon in der Wärme schied sich aus der tiefblauen Flüssigkeit ein blauer, deutlich von dem überschüssigen Hydroxyd unterscheidbarer Niederschlag ab. Nach dem Erkalten wurde dieser Niederschlag abfiltrirt und ausgewaschen. Es fiel auf, dass er nicht die schön sattblaue Färbung des erwarteten Leucinkupfers hatte, sondern graublau gefärbt war. Mikroskopisch bestand er aus stark lichtbrechenden, blassblau gefärbten Kugeln. Eine Probe des Niederschlags war in kaltem Wasser unlöslich, in viel Salzsäure löste sie sich.

Die Hauptmenge des Niederschlages wurde in Wasser suspendirt und nach Zusatz einiger Tropfen Salzsäure durch Schwefelwasserstoff in der Wärme zerlegt, der Schwefelwasserstoff durch Kochen verjagt. Dabei fiel Cystin in Form von schön durchsichtigen, sechsseitigen Tafeln aus.

Es konnte sich sonach nur um das noch nicht beschriebene Kupfersalz des Cystins gehandelt haben. ${ }^{1}$ ) In der That erhält man beim Kochen von Cystin in wässeriger Suspension oder Lösung mit Cuprihydroxyd sehr leicht die hellgraublaue Kupferverbindung. Dieselbe krystallisirt in verschiedenen Formen; ich beobachtete einmal sechsseitige Täfelchen, die, von der blauen Farbe abgesehen, denen des Cystins völlig glichen, ein anderes Mal lange, schön ausgebildete Nadeln.

\section{Nachweis von Oystein und Oystin unter den Spaltungs- produkten des Fieralbumins.}

Meine Versuche, aus echten Eiweissstoffen Cystin darzustellen, erstreckten sich auf Serumeiweiss und Eiereiweiss, von denen namentlich das erstere durch seinen grossen Gehalt an abspaltbarem Schwefel ${ }^{2}$ ) von vornherein als besonders geeignet zu unsern Versuchen erschien. Ein vorläufiger Versuch wurde ferner mit krystallisirtem Edestin aus Hanfsamen angestellt.

1) Hier muss Mörner's Angabe erwähnt werden, dass eine durch Kochen mit Wasser bereitete Cystinlösung mit Kupferacetat eine geringe Fällung gab.

2) Siehe Fr. N. Schulz, Diese Zeitschrift, Bd. XXV, S. 30. 
Zunächst wandte ich dieselbe Methode wie vorher bei der Hornsubstanz an. Die durch 5 stündiges Sieden des Eiweisskörpers (Serumalbumin) mit concentrirter Salzsäure erhaltene Zersetzungsflüssigkeit wurde in der oben beschriebenen Weise neutralisirt, bei schwach salzsaurer Reaction mit Thierkohle entfärbt und auf dem Wasserbade eingeengt. Keine der bei salzsaurer Reaction erhaltenen Krystallisationsfractionen enthielt nach dem Auswaschen mit kaltem Wasser erhebliche Mengen abspaltbaren Schwefels. Der letztere war vielmehr immer nur in den Mutterlaugen nachweisbar.

Es liess sich also kein Cystin durch fractionirte Krystallisation gewinnen und die Untersuchung richtete sich nunmehr auf das Vorhandensein von Cystein.

Für die Gewinnung von Cystein ist eine Reihe von Fällungsmethoden angegeben worden. Brenzinger ${ }^{1}$ ) fand, dass das salzsaure Cystein mit Quecksilberchlorid in wässeriger Lösung eine fast ganz unlösliche Verbindung gibt. Boris sow ${ }^{2}$ ) reducirte durch Behandeln mit Zink und Salzsäure das bei Cystinurie im Harn vorhandene Cystin zu Cystein und fällte diesen Körper ebenfalls durch Sublimat (unter Zusatz von Natriumacetat). Suter ${ }^{3}$ ) versuchte vergeblich, aus von Hornsubstanz stammenden Tyrosinmutterlaugen unter Verwendung der Fällung mit Sublimat Cystein resp. Cystin zu gewinnen. Derselbe Autor ${ }^{4}$ ) stellte durch Schütteln mit Benzylchlorid und Natronlauge eine in kaltem Wasser wenig lösliche Benzylverbindung des Cysteins her. Ein Versuch, durch Benzyliren Cystein aus einer Tyrosinmutterlauge zu gewinnen, misslang. ${ }^{5}$ ) Mörner ${ }^{6}$ ) verwandte in seiner öfters erwähnten ersten Arbeit wiederum Sublimat sowie Mercuriacetat zur Ausfällung des Cysteins, wobei er zur Abstumpfung der sauren Reaction Natronlauge hinzufügte. Es gelang ihm, auf diese Weise die

1) Diese Zeitschrift, Bd. XVI, S. 557.

2) Diese Zeitschrift, Bd. XIX, S. 511.

3) Diese Zeitschrift, Bd. XX, S. 564 .

4) Diese Zeitschrift, Bd. XX, S. 562 .

5) Diese Zeitschrift, Bd. XX, S. 576.

6) l. c., S. 612 . 
bleischwärzende Substanz nahezu vollständig in den Quecksilberniederschlag $\mathrm{zu}$ bringen. Einen Theil des Cysteins konnte er auch durch Kupferacetat unter Vermeidung eines Ueberschusses ausfällen.

Bei der vorliegenden Untersuchung wurde auf zweierlei Weise versucht, das Vorhandensein von Cystein unter den Spaltungsprodukten von genuinen Eiweisssubstanzen zu erweisen.

Erstens bemühte ich mich, das Cystein in der Mutterlauge durch Zusatz von Oxydationsmitteln (Eisenchlorid in verschiedenen Mengen und Wasserstoffsuperoxyd) zu Cystin $\mathrm{zu}$ oxydiren, um das letztere durch fractionirte Krystallisation zu gewinnen. Zweitens benutzte auch ich die von Brenzinger herrührende und von Suter, Borissow und Mörner ${ }^{1}$ ) angewandte Quecksilbermethode. Ich wandte vorwiegend Sublimat, in vereinzelten Fällen auch Mercuriacetat an.

Die zahlreichen Versuche, die zu keinem endgültigen Resultat führten, sollen einstweilen ausser Acht gelassen werden; es sei zunächst kurz über den Versuch berichtet, der schliesslich zum Ziele führte:

$500 \mathrm{~g}$ käufliches Eiereiweiss werden mit $1500 \mathrm{ccm}$. reiner Salzsäure vom specifischen Gewicht 1,124 durch $8-81 / 2$ Stunden auf dem Sandbade zum Sieden erhitzt, nach dem Abkühlen mit Natronlauge unter Vermeiden von stärkerem Erwärmen versetzt, mehrere Tage stehen gelassen und vom entstandenen Niederschlage abfiltrirt; zum amphoter reagirenden Filtrat werden ohne vorhergehende Entfärbung mit Thierkohle ca. $500 \mathrm{~g}$ Quecksilberchlorid in warmer, wässeriger Lösung (doch unter Vermeidung von stärkerem Erwärmen der Mutterlauge) hinzugefügt; es tritt auch nach einigen Stunden nur eine mässig starke Trübung auf; auf Zusatz einer nicht unerheblichen Menge Natronlauge (bis zur deutlich alkalischen Reaction) tritt ein reichlicher, flockiger, braungefärbter Niederschlag auf, der sich ziemlich rasch zu Boden setzt. Am nächsten Tage wird dieser Niederschlag abgetrennt, $z u$ wiederholten Malen mit reichlichen Mengen kalten Wassers decantirt, auf einem Büchner'schen Filter gut abgesaugt, in etwa $600 \mathrm{ccm}$. Wasser ohne Sạurezusatz suspendirt und mit Schwefelwasserstoff in der Kälte zersetzt. Die vom Sulfidniederschlag abfiltrirte, deutlich sauer reagirende, gelbe Flüssigkeit

1) 1. c., S. 611 . 
wird nach Zusatz einiger Tropfen concentrirter Salzsäure durch Sieden vom Schwefelwasserstoff befreit. Sie gibt jetzt folgende Reactionen:

1. Mit Natronlauge und Bleiacetat allmählich eintretende starke Schwefelbleireaction.

2. Mit Eisenchlorid (nachdem die Flüssigkeit zur möglichsten Beseitigung der störenden Gelbfärbung stark mit Wasser verdünnt war) rasch verschwindende Indigoblaufärbung.

3. Mit Natronlauge und Nitroprussidnatrium purpurrothe, bald in Gelb übergehende Färbung.

4. Mit Kupfersulfat intensive, violette, sofort verblassende Färbung, dann Bildung eines graublauen Niederschlags.

5. Bei vorsichtigem Zusatz von Ammoniak eine violettrothe Färbung.

(Diese Reaction ist von Andreasch1) für Cysteinlösungen bei Gegenwart von Eisen angegeben worden. Die Färbung verschwindet, wenn man die Flüssigkeit ruhig im Reagensglase stehen lässt, nach einigen Minuten, um beim Schütteln mit Luft wiederzukehren und abermals zu verschwinden. Durch Wiederholung des Schüttelns kann man sie dann noch ein- oder mehrere Male in allmählich schwächer: werdendem Grade hervorrufen. Aehnliches gibt Andreasch an.)

Die Flüssigkeit wurde jetzt ammoniakalisch gemacht, und während ca. 24. Stunden ein langsamer Luftstrom hindurchgeleitet. Die Eisenchlorid- und die Kupfersulfatreaction sind (selbstverständlich nach voraufgehendem Neutralisiren der Flüssigkeit) schon nach kurzer Zeit nicht mehr deutlich hervorzurufen. Die Nitroprussidreaction ist nach einigen Stunden sehr viel schwächer als vorher, nach 24 Stunden verschwunden.

Ein kleiner Theil der Flüssigkeit wird über concentrirte Schwefelsäure in den Vacuumexsiccator gebracht. Ueber Nacht ist eine kleine Menge von zumeist tyrosinähnlichen Krystallen ausgefallen, die leicht in Ammoniak, sehr schwer in kaltem Wasser löslich sind, aber keine Millon'sche Reaction geben.

Es wird nunmehr die ganze noch vorhandene Flüssigkeit (der Haupttheil war zu anderweitigen Cystindarstellungsversuchen aufgebraucht worden) bei salzsaurer Reaction möglichst rasch auf dem Wasserbad bis auf etwa $40 \mathrm{ccm}$. eingedampft. Der ziemlich dickflüssige und dunkelbraune Rückstand wurde jetzt ganz schwach ammoniakalisch gemacht, hierauf mit Eisessig bis zur eben sauren Reaction versetzt und auf acht Tage in den Vacuumexsiccator gebracht. Es bildete sich bald eine Trübung, dann ein Niederschlag, der während der nächsten Tage langsam zunahm. Mikroskopisch bestand er aus undurchsichtigen Kugeln, neben amorphen Partikeln.

Nach acht Tagen wurde er abfiltrirt, mehrfach mit kaltem Wasser ausgewaschen, dann in einer geringen Menge verdünnten Ammoniaks

1) Maly's Jahresbericht über die Fortschritte der Thierchemie, Bd. 14, 1885, S. 76, Anmerkung. 
gelöst, durch vorsichtiges Ansäuern mit Essigsäure wieder ausgefällt und neuerdings so lange gewaschen, bis das ablaufende Waschwasser keine Reaction mit Nessler's Reagens gab.

Der so gewonnene Körper war noch etwas gefärbt; er erwies sich mikroskopisch als durchweg krystallinisch. Die Krystalle hatten in der Hauptmasse die für das Cystin bekannte Form von sechsseitigen Tafeln, daneben fanden sich auch in geringer Menge rhombische Krystalle vor. Die Substanz enthielt reichlich abspaltbaren Schwefel. Eine Stickstoffbestimmung nach Dumas ${ }^{1}$ ) ergab folgendes Resultat:

$0,0906 \mathrm{~g}$ der Substanz lieferten bei $23^{\circ}$ und einem auf Trockenheit reducirten Barometerstand von $740 \mathrm{~mm} \mathrm{9,7} \mathrm{ccm} . \mathrm{N}=0,01059 \mathrm{~g} \mathrm{~N}=$ $11,68 \% \mathrm{~N}$ (statt der für Cystin berechneten 11,69\% N).

Nach allem diesen kann es keinem Zweifel unterliegen, dass die vorliegende Substanz Cystin war, und dass die Zersetzungsflüssigkeit des Quecksilberniederschlages Cystein enthielt, dass also das Cystein ein Spaltungsprodukt des Eieralbumins ist.

Wie beim Eieralbumin gelang es auch beim Serumalbumin und Edestin, nach Zersetzung mit Salzsäure und Quecksilberfällung Cysteinreactionen $\mathrm{zu}$ erhalten. Beim Serumalbumin gelangte ich in einem Versuche auch $\mathrm{zu}$ krystallinischem Cystin.

Hält man daneben die oben angeführten Befunde Mörner's, so gewinnt es den Anschein, dass jene Eiweissstoffe, welche leicht abspaltbaren Schwefel aufweisen, regelmässig in ihrem Molekül die Cysteingruppe enthalten. Wenn Mörner für die Keratinsubstanzen der Meinung zuneigt, dass sie bereits die Dithioverbindung des Cysteins, das Cystin, enthalten, so kann ich ihm in Hinblick auf die Leichtigkeit, mit der sie bei Spaltung fertiges Cystin liefern, nur beipflichten. Bei den schwefelarmen Eiweissstoffen scheint sich die Sache anders zu verhalten, da hier, wie oben erwähnt, bei kurzdauernder Spaltung nur Cystein auftritt.

1) Für die liebenswürdige Ausführung dieser Bestimmung spreche ich auch an dieser Stelle Herrn Hassler, Hülfsarbeiter am chemischen Staatslaboratorium in Hamburg, meinen verbindlichsten Dank aus. 